TRANSACTIONS OF THE

AMERICAN MATHEMATICAL SOCIETY

Volume 358, Number 1, Pages 1-10

S 0002-9947(05)03907-3

Article electronically published on August 25, 2005

\title{
HOMOTOPICAL VARIATIONS AND HIGH-DIMENSIONAL ZARISKI-VAN KAMPEN THEOREMS
}

\author{
D. CHÉNIOT AND C. EYRAL
}

\begin{abstract}
We give a new definition of the homotopical variation operators occurring in a recent high-dimensional Zariski-van Kampen theorem, a definition which opens the way to further generalizations of theorems of this kind.
\end{abstract}

\section{INTRODUCTION}

The classical Zariski-van Kampen theorem on curves gives a presentation by generators and relations of the fundamental group of the complement of an algebraic curve in the complex projective plane (cf. [Za, $\mathrm{VK}]$ and [C1]). There exist high-dimensional analogues of this theorem describing relevant higher-homotopy groups of complements of algebraic hypersurfaces with isolated singularities (cf. Li] and (CL). These theorems use 'degeneration operators' or 'homotopical variation operators' which are not so easy to define. The definitions go through the homology of universal covers, and the link between homotopy and homology depends strongly on the special topology of the complements of hypersurfaces with isolated singularities. The aim of this note is to give a very short and purely homotopical definition of the homotopical variation operators. Moreover, our definition makes sense in a very general setting, that of a possibly singular quasi-projective variety, and thus opens the way to obtain more general Zariski-van Kampen theorems (see our conjecture at the end of Section 4).

The content of the article is as follows. Section 1 is devoted to some basic facts on generic pencils and monodromies which are essential ingredients in theorems of Zariski-van Kampen type. In Section 2, we recall the definition of the homological variation operators $\operatorname{var}_{i, q}$ of $\mathrm{C} 3$ which are used to define the homotopical variation operators $\mathcal{V} \mathcal{A R}_{i, n-1}$ of [CL]. The latter will be described in Section 3 . In Sections 4 and 5 , we introduce our generalized homotopical variation operators $\mathrm{VAR}_{i, q}$, we give their elementary properties and we prove that they coincide with those of CL when the latter are defined.

\section{NotATION}

Throughout the paper, homology groups are singular homology groups with integer coefficients, unless there is an explicit statement to the contrary. We shall note the homology class in a space $A$ of an (absolute) cycle $z$ by $[z]_{A}$ and the

Received by the editors December 9, 2002.

2000 Mathematics Subject Classification. Primary 14F35; Secondary 14D05, 32S50, 55Q99.

Key words and phrases. Homotopy groups of algebraic varieties, pencils of hyperplanes, monodromies. 
homology class in $A$ modulo a subspace $B$ of a relative cycle $z^{\prime}$ by $\left[z^{\prime}\right]_{A, B}$. If there is no ambiguity, we shall omit the subscripts. If $(A, B)$ is a pointed pair with base point $* \in B$, we shall denote by $F^{q}(A, B, *)$ the set of relative homotopy $q$-cells of $A$ modulo $B$ based at $*$. These are maps from the $q$-cube $I^{q}$ to $A$, with the face $x_{q}=0$ sent into $B$ and all other faces sent to $*$ (as in [St, Section 15]). We denote by $F^{q}(A, *)$ the set of absolute homotopy $q$-cells of $A$ based at $*$, that is, maps from $I^{q}$ to $A$ sending the boundary $\dot{I}^{q}$ of $I^{q}$ to $*$. Given $f \in F^{q}(A, B, *)\left(\right.$ resp. $\left.F^{q}(A, *)\right)$, the homotopy class of $f$ in $A$ modulo $B$ based at $*$ (resp. in $A$ based at $*$ ) will be denoted by $\langle f\rangle_{A, B, *}\left(\right.$ resp. $\left.\langle f\rangle_{A, *}\right)$. Again, if there is no ambiguity, we shall omit the subscripts.

\section{Generic Pencils And MOnodromies}

Let $X:=Y \backslash Z$, where $Y$ is a non-empty (closed) algebraic subset of $\mathbb{C P}^{n}$, with $n \geq 2$, and $Z$ is a proper algebraic subset of $Y$. The quasi-projective variety $X$ may have singularities. Take a Whitney stratification $\mathcal{S}$ of $Y$ such that $Z$ is a union of strata (cf. Wh, $\left[\mathrm{LT}\right.$ ), and consider a projective hyperplane $\mathcal{L}$ of $\mathbb{C P}^{n}$ transverse to (the strata of) $\mathcal{S}$ (the choice of such a hyperplane is generic).

Let $\mathcal{P}$ be a pencil of hyperplanes of $\mathbb{C P}^{n}$ having $\mathcal{L}$ as a member and whose axis $\mathcal{M}$ is transverse to $\mathcal{S}$ (the choice of such an axis is generic inside $\mathcal{L}$ ). All the members of $\mathcal{P}$ are transverse to $\mathcal{S}$ with the exception of a finite number of them $\left(\mathcal{L}_{i}\right)_{i}$, called exceptional hyperplanes, for which, nevertheless, there are only a finite number of points of non-transversality, all of them situated outside of $\mathcal{M}$ (cf. [C2, Section 10]). If necessary, one may take the liberty of considering some ordinary members of $\mathcal{P}$, different from $\mathcal{L}$, as exceptional ones.

We parametrize the elements of $\mathcal{P}$ by the complex projective line $\mathbb{C P}^{1}$ as usual. Let $\lambda$ be the parameter of $\mathcal{L}$ and, for each $i$, let $\lambda_{i}$ be the parameter of $\mathcal{L}_{i}$. For each $i$, take a small closed disk $D_{i} \subset \mathbb{C P}^{1}$ with centre $\lambda_{i}$ together with a point $\gamma_{i}$ on its boundary. Choose the $D_{i}$ mutually disjoint. Also take the image $\Gamma_{i}$ of a simple arc in $\mathbb{C P}^{1}$ joining $\lambda$ to $\gamma_{i}$ and such that: (i) $\Gamma_{i} \cap D_{i}=\gamma_{i}$; (ii) $\Gamma_{i} \cap \Gamma_{i^{\prime}}=\lambda$ if $i \neq i^{\prime}$; (iii) $\Gamma_{i} \cap D_{i^{\prime}}=\emptyset$ if $i \neq i^{\prime}$. Then, set

$$
K_{i}:=\Gamma_{i} \cup D_{i} .
$$

Finally, consider a loop $\omega_{i}$ in the boundary $\partial K_{i}$ of $K_{i}$ starting from $\lambda$, running along $\Gamma_{i}$ up to $\gamma_{i}$, going once counter-clockwise around the boundary of $D_{i}$ and coming along $\Gamma_{i}$ back to $\lambda$.

For any subsets $G \subset \mathbb{C P}^{n}$ and $E \subset \mathbb{C P}^{1}$, note $G_{E}:=\bigcup_{\mu \in E} G \cap \mathcal{P}(\mu)$, where $\mathcal{P}(\mu)$ is the member of $\mathcal{P}$ with parameter $\mu$.

Lemma 1.1 (cf. [C3, Lemma 4.1]). For each $i$, there is an isotopy

$$
H:(\mathcal{L} \cap X) \times I \rightarrow X_{\partial K_{i}}
$$

such that:

(i) $H(x, 0)=x$, for every $x \in \mathcal{L} \cap X$;

(ii) for every fixed $t \in I, H$ induces a homeomorphism from $\mathcal{L} \cap X$ onto $X_{\omega_{i}(t)}$;

(iii) $H(x, t)=x$, for every $x \in \mathcal{M} \cap X$ and every $t \in I$.

As usual, $I$ is the unit interval $[0,1]$.

The terminal homeomorphism

$$
h: \mathcal{L} \cap X \rightarrow \mathcal{L} \cap X
$$


of $H$, defined by $h(x):=H(x, 1)$, of course leaves $\mathcal{M} \cap X$ pointwise fixed. Such a homeomorphism $h$ is called a geometric monodromy of $\mathcal{L} \cap X$ relative to $\mathcal{M} \cap X$ above $\omega_{i}$.

Another choice of loop $\omega_{i}$ within the same homotopy class

$$
\left\langle\omega_{i}\right\rangle \in \pi_{1}\left(\mathbb{C P}^{1} \backslash \bigcup_{i} \lambda_{i}, \lambda\right)
$$

and another choice of isotopy $H$ above $\omega_{i}$ as in Lemma 1.1 would give a geometric monodromy isotopic to $h$ within $\mathcal{L} \cap X$ by an isotopy leaving $\mathcal{M} \cap X$ pointwise fixed. Thus, the isotopy class of $h$ in $\mathcal{L} \cap X$ relative to $\mathcal{M} \cap X$ is wholly determined by the homotopy class $\left\langle\omega_{i}\right\rangle \in \pi_{1}\left(\mathbb{C P}^{1} \backslash \bigcup_{i} \lambda_{i}, \lambda\right)$.

\section{HomologicAl VARIATION OPERATORS}

Fix an index $i$, and consider a geometric monodromy $h$ of $\mathcal{L} \cap X$ relative to $\mathcal{M} \cap X$ above $\omega_{i}$. Denote by $S_{q}(\mathcal{L} \cap X)$ the abelian group of singular $q$-chains of $\mathcal{L} \cap X$ with integer coefficients, and by $h_{q}: S_{q}(\mathcal{L} \cap X) \rightarrow S_{q}(\mathcal{L} \cap X)$ the chain homomorphism induced by $h$. Since $h$ leaves $\mathcal{M} \cap X$ pointwise fixed (cf. Lemma 1.1), it is easy to see that for every relative $q$-cycle $z$ of $\mathcal{L} \cap X$ modulo $\mathcal{M} \cap X$, the variation by $h_{q}$ of $z$, that is, the chain $h_{q}(z)-z$, is an absolute $q$-cycle of $\mathcal{L} \cap X$ (cf. [C3, Lemma 4.6]). Moreover, one has the following lemma.

Lemma 2.1 (cf. [C3, Lemma 4.8]). The correspondence

$$
\begin{aligned}
\operatorname{var}_{i, q}: H_{q}(\mathcal{L} \cap X, \mathcal{M} \cap X) & \rightarrow H_{q}(\mathcal{L} \cap X) \\
{[z]_{\mathcal{L} \cap X, \mathcal{M} \cap X} } & \mapsto\left[h_{q}(z)-z\right]_{\mathcal{L} \cap X}
\end{aligned}
$$

gives a well-defined homomorphism which depends only on the homotopy class $\left\langle\omega_{i}\right\rangle \in \pi_{1}\left(\mathbb{C P}^{1} \backslash \bigcup_{i} \lambda_{i}, \lambda\right)$.

Homomorphism $\operatorname{var}_{i, q}$ is called a homological variation operator associated to $\left\langle\omega_{i}\right\rangle$. It is analogous to the classical variation operator associated with a holomorphic function having isolated critical points (cf. AGV]). Note that operator $\operatorname{var}_{i, q}$ is defined even if $X$ has singularities.

\section{ChÉniot-Libgober's HOMOTOPICAL VARIATION OPERATORS}

Throughout this section, we work under the following hypotheses.

Hypotheses 3.1. We assume that $Y=\mathbb{C P}^{n}$, with $n \geq 3$, and $Z=H$, where $H$ is a (closed) algebraic hypersurface of $\mathbb{C P}^{n}$, with degree $k$, having only isolated singularities. Thus, $X=\mathbb{C P}^{n} \backslash H$. We also assume that $\mathcal{S}$ is the Whitney stratification the strata of which are: $\mathbb{C P}^{n} \backslash H$, the singular part $H_{\text {sing }}$ of $H$, and the non-singular part $H \backslash H_{\text {sing }}$ of $H$. Being transverse to $\mathcal{S}$ then means avoiding the singularities of $H$ and being transverse to the non-singular part of $H$. Observe that $\mathcal{M} \cap X \neq \emptyset$. We fix a base point $* \in \mathcal{M} \cap X$.

In [CL], a $k$-fold (unramified) holomorphic covering

$$
p: \quad X^{\prime} \rightarrow X
$$

is constructed, where $X^{\prime}:=Y^{\prime} \backslash Z^{\prime}$ is a (pathwise) connected quasi-projective variety in $\mathbb{C P}^{n+1}$. In fact, $X^{\prime}$ is the global Milnor fibre of the cone of $\mathbb{C}^{n+1}$ corresponding to $H$. Moreover, it is shown that there is a Whitney stratification $\mathcal{S}^{\prime}$ 
of $Y^{\prime}$ preserving $Z^{\prime}$ and a pencil $\mathcal{P}^{\prime}$ in $\mathbb{C} P^{n+1}$ with axis $\mathcal{M}^{\prime}$ transverse to $\mathcal{S}^{\prime}$ such that

$$
\begin{aligned}
p^{-1}(\mathcal{M} \cap X) & =\mathcal{M}^{\prime} \cap X^{\prime} & & \text { and } \\
p^{-1}(\mathcal{P}(\mu) \cap X) & =\mathcal{P}^{\prime}(\mu) \cap X^{\prime} & & \text { for every } \mu \in \mathbb{C P}^{1},
\end{aligned}
$$

the member $\mathcal{P}^{\prime}(\mu)$ of $\mathcal{P}^{\prime}$ with parameter $\mu$ being transverse to $\mathcal{S}^{\prime}$ if and only if $\mathcal{P}(\mu)$ is transverse to $\mathcal{S}$. Recall that $\mathcal{L}=\mathcal{P}(\lambda)$, and put $\mathcal{L}^{\prime}:=\mathcal{P}^{\prime}(\lambda)$. Then, for each $i$, the pencil $\mathcal{P}^{\prime}$ gives rise to a homological variation operator

$$
\operatorname{var}_{i, n-1}^{\prime}: H_{n-1}\left(\mathcal{L}^{\prime} \cap X^{\prime}, \mathcal{M}^{\prime} \cap X^{\prime}\right) \rightarrow H_{n-1}\left(\mathcal{L}^{\prime} \cap X^{\prime}\right)
$$

associated to $\left\langle\omega_{i}\right\rangle$, defined as in Section 2 .

Given an index $i$ and a base point $\bullet \in p^{-1}(*)$, one can then consider the following diagram:

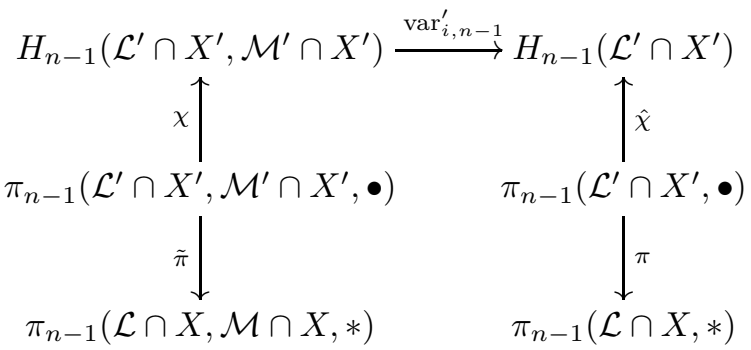

where $\tilde{\pi}$ and $\pi$ are induced by $p$ and where $\chi$ and $\hat{\chi}$ are Hurewicz homomorphisms. Now, by a general property of covering projections, $\tilde{\pi}$ is an isomorphism (cf. Sp, Theorem 7.2.8]). Moreover, homomorphism $\hat{\chi}$, too, is an isomorphism as a consequence of the special fact that $X$ is the complement of a projective hypersurface $H$ with isolated singularities. Indeed, $\mathcal{L} \cap H$ is then a non-singular hypersurface of $\mathcal{L} \simeq \mathbb{C P}^{n-1}$, so that

$$
\pi_{1}(\mathcal{L} \cap X, *) \simeq \mathbb{Z} / k \mathbb{Z} \quad \text { and } \quad \pi_{q}(\mathcal{L} \cap X, *)=0 \quad \text { for } \quad 2 \leq q \leq n-2
$$

(this range may be empty) (cf. [Li, Lemma 1.1]). Knowing that $\mathcal{L}^{\prime} \cap X^{\prime}$ is pathwise connected (cf. CL, Lemma 2.9]), these facts imply that $\mathcal{L}^{\prime} \cap X^{\prime}$ is $(n-2)$-connected, and $\hat{\chi}$ is then an isomorphism by the Hurewicz isomorphism theorem.

Thus, for each $i$, and for every $\bullet \in p^{-1}(*)$, there is a homomorphism

$$
\mathcal{V} \mathcal{A R}_{i, n-1}: \pi_{n-1}(\mathcal{L} \cap X, \mathcal{M} \cap X, *) \rightarrow \pi_{n-1}(\mathcal{L} \cap X, *)
$$

defined by the composition $\pi \circ \hat{\chi}^{-1} \circ \operatorname{var}_{i, n-1}^{\prime} \circ \chi \circ \tilde{\pi}^{-1}$ in diagram (3.2) (cf. CLL, Section 5]).

It will easily follow from Section 5 that homomorphism $\mathcal{V} \mathcal{A} \mathcal{R}_{i, n-1}$ does not in fact depend on the choice of the base point $\bullet \in p^{-1}(*)$.

Homomorphism $\mathcal{V} \mathcal{A} \mathcal{R}_{i, n-1}$ is called a homotopical variation operator associated to $\left\langle\omega_{i}\right\rangle$.

\section{Generalized homotopical Variation operators}

In this section, $X:=Y \backslash Z$ is again a (possibly singular) quasi-projective variety as in Sections 1 and 2. We further assume that $\mathcal{M} \cap X \neq \emptyset$ and we fix a base point $*$ in $\mathcal{M} \cap X$. Observe that the condition $\mathcal{M} \cap X \neq \emptyset$ is equivalent to $\operatorname{dim} X \geq 2$. We also fix an index $i$, and consider a geometric monodromy $h$ of $\mathcal{L} \cap X$ relative to $\mathcal{M} \cap X$ above $\omega_{i}$ (cf. Section 1$)$. 
Let $q$ be an integer $\geq 1$ and let $f \in F^{q}(\mathcal{L} \cap X, \mathcal{M} \cap X, *)$. We consider the map $f \perp(h \circ f)$ defined on $I^{q}:=[0,1]^{q}$ by

$$
f \perp(h \circ f)\left(x_{1}, \ldots, x_{q}\right):=\left\{\begin{array}{c}
f\left(x_{1}, \ldots, x_{q-1}, 1-2 x_{q}\right), \quad 0 \leq x_{q} \leq \frac{1}{2}, \\
h \circ f\left(x_{1}, \ldots, x_{q-1}, 2 x_{q}-1\right), \quad \frac{1}{2} \leq x_{q} \leq 1 .
\end{array}\right.
$$

Since $h$ leaves $\mathcal{M} \cap X$ pointwise fixed, this map is well defined and belongs to $F^{q}(\mathcal{L} \cap X, *)$. Note that the reversion of $f$ and its concatenation with $h \circ f$ are performed on the variable transverse to the free face. This would in general not make sense, but here it does because $f$ and $h \circ f$ have the same boundary.

Lemma 4.1. The correspondence

$$
\begin{aligned}
\operatorname{VAR}_{i, q}: \pi_{q}(\mathcal{L} \cap X, \mathcal{M} \cap X, *) & \rightarrow \pi_{q}(\mathcal{L} \cap X, *) \\
\langle f\rangle_{\mathcal{L} \cap X, \mathcal{M} \cap X, *} & \mapsto\langle f \perp(h \circ f)\rangle_{\mathcal{L} \cap X, *}
\end{aligned}
$$

gives a well-defined map which depends only on the homotopy class

$$
\left\langle\omega_{i}\right\rangle \in \pi_{1}\left(\mathbb{C P}^{1} \backslash \bigcup_{i} \lambda_{i}, \lambda\right) .
$$

If $q \geq 2$, it is a homomorphism.

Proof. If $\left(H_{t}\right)_{t \in I}$ is a homotopy between two representatives $f$ and $g$ of the same element of $\pi_{q}(\mathcal{L} \cap X, \mathcal{M} \cap X, *)$, then $\left(H_{t} \perp\left(h \circ H_{t}\right)\right)_{t \in I}$ is a homotopy based at $*$ between $f \perp(h \circ f)$ and $g \perp(h \circ g)$, showing that they belong to the same element of $\pi_{q}(\mathcal{L} \cap X, *)$. Thus, the considered map is well defined. One easily checks that it is a homomorphism if $q \geq 2$, the sum of homotopy cells being performed as in $\mathrm{St}$, Section 15]. The independence assertion follows from the remark we made just after Lemma 1.1.

We shall call map $\operatorname{VAR}_{i, q}$ a generalized homotopical variation operator associated to $\left\langle\omega_{i}\right\rangle$. This terminology is justified by Theorem 5.1 below which asserts that, in the case where the homotopical variation operators of $\mathrm{CL}$ are defined (cf. Section $3)$, the latter coincide with our generalized operators.

We remark that if our operators are applied to absolute cells of $\mathcal{L} \cap X$ or if their result is considered as relative cells of $\mathcal{L} \cap X$ modulo $\mathcal{M} \cap X$, then they act as what can be called ordinary variations by monodromy. More precisely:

Observation 4.2. Let $\operatorname{incl}_{q}: \pi_{q}(\mathcal{L} \cap X, *) \rightarrow \pi_{q}(\mathcal{L} \cap X, \mathcal{M} \cap X, *)$ be the natural map. Then:

(i) $\operatorname{VAR}_{i, q}\left(\operatorname{incl}_{q}(x)\right)=-x+h_{q}(x)$ for all $x \in \pi_{q}(\mathcal{L} \cap X, *)$;

(ii) if $q \geq 2$, $\operatorname{incl}_{q}\left(\operatorname{VAR}_{i, q}(y)\right)=-y+\tilde{h}_{q}(y)$ for all $y \in \pi_{q}(\mathcal{L} \cap X, \mathcal{M} \cap X, *)$;

where $h_{q}$ and $\tilde{h}_{q}$ are the automorphisms of $\pi_{q}(\mathcal{L} \cap X, *)$ and $\pi_{q}(\mathcal{L} \cap X, \mathcal{M} \cap X, *)$ respectively induced by $h$.

The right-hand sides of the equalities are written additively though the first group is not a priori commutative if $q=1$ nor is the second one if $q=2$; the order of operations must then be respected.

Observation 4.2 relies on the same reasons as those which allow the sum of two homotopy cells to be performed indiscriminately on any variable not transverse to the (possible) free face and which make the sum commutative in high dimension. Its detailed proof is left to the reader. 
Operator $\operatorname{VAR}_{i, q}$ is linked to the homological variation operator $\operatorname{var}_{i, q}$ of Section 2 by Hurewicz homomorphisms. This is stated in the next lemma.

Lemma 4.3. The following diagram is commutative:

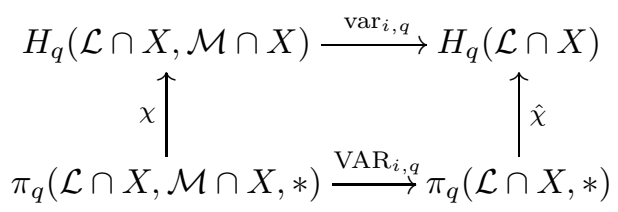

where $\chi$ and $\hat{\chi}$ are Hurewicz homomorphisms.

Proof. Since homotopy cells are defined on cubes, it is convenient to use cubical singular homology theory (cf. [HW], $[\mathrm{M}]$ ), which is equivalent to ordinary (simplicial) singular theory (cf. HW] Section 8.4]). So, let us first introduce some notation. For any pair of spaces $(U, V)$, with $V \subset U$, we shall denote by $H_{q}^{c}(U, V)$ the $q$-th cubical singular relative homology group of $(U, V)$, by $[\cdot]_{U, V}^{c}$ the homology classes in this group, and by $S_{q}^{c}(U, V)$ the (normalized) group of $q$-dimensional cubical chains of the pair $(U, V)$. We shall freely use representatives of the elements of $S_{q}^{c}(U, V)$. Given a (continuous) map $g:(U, V) \rightarrow\left(U^{\prime}, V^{\prime}\right)$, we shall denote by $g_{q}: S_{q}^{c}(U, V) \rightarrow S_{q}^{c}\left(U^{\prime}, V^{\prime}\right)$ the induced cubical chain homomorphism. A similar notation is used for the absolute case.

Let $f \in F^{q}(\mathcal{L} \cap X, \mathcal{M} \cap X, *)$ be a representative of an element of

$$
\pi_{q}(\mathcal{L} \cap X, \mathcal{M} \cap X, *) .
$$

We have

$$
\begin{aligned}
\chi\left(\langle f\rangle_{\mathcal{L} \cap X, \mathcal{M} \cap X, *}\right) & =\left[f_{q}(\iota)\right]_{\mathcal{L} \cap X, \mathcal{M} \cap X}^{c}, \\
\hat{\chi}\left(\langle f \perp(h \circ f)\rangle_{\mathcal{L} \cap X, *}\right) & =\left[(f \perp(h \circ f))_{q}(\iota)\right]_{\mathcal{L} \cap X}^{c},
\end{aligned}
$$

where $\iota: I^{q} \rightarrow I^{q}$ is the identity map (cf. [HW, 8.8.4]). Since the expression for $\operatorname{var}_{i, q}$ (given by Lemma 2.1) remains valid in cubical theory (by [HW] 8.4.7 and the paragraph before 8.4.10]), it then suffices to prove that the following equality holds in $H_{q}^{c}(\mathcal{L} \cap X)$ :

$$
\left[h_{q}\left(f_{q}(\iota)\right)-f_{q}(\iota)\right]_{\mathcal{L} \cap X}^{c}=\left[(f \perp(h \circ f))_{q}(\iota)\right]_{\mathcal{L} \cap X}^{c} .
$$

For this purpose, consider the singular $q$-cubes $\sigma_{1}, \sigma_{2}: I^{q} \rightarrow I^{q}$ in $I^{q}$ defined by

$$
\begin{aligned}
& \sigma_{1}\left(x_{1}, \ldots, x_{q}\right):=\left(x_{1}, \ldots, x_{q-1}, \frac{1-x_{q}}{2}\right), \\
& \sigma_{2}\left(x_{1}, \ldots, x_{q}\right):=\left(x_{1}, \ldots, x_{q-1}, \frac{1+x_{q}}{2}\right) .
\end{aligned}
$$

Observe that $-\sigma_{1}+\sigma_{2}$ is a relative cycle of $I^{q}$ modulo $\dot{I}^{q}$.

Lemma 4.5. The following equality holds in $H_{q}^{c}\left(I^{q}, \dot{I}^{q}\right)$ :

$$
\left[-\sigma_{1}+\sigma_{2}\right]_{I^{q}, \dot{I}^{q}}^{c}=[\iota]_{I^{q}, \dot{I}^{q}}^{c} .
$$

Proof. Let $\sigma: I^{q+1} \rightarrow I^{q}$ be the singular $(q+1)$-cube in $I^{q}$ defined by

$$
\sigma\left(x_{1}, \ldots, x_{q+1}\right):=\left\{\begin{array}{lr}
\left(x_{1}, \ldots, x_{q-1}, \frac{2 x_{q+1}+x_{q}-1}{2}\right), & x_{q+1} \geq-\frac{x_{q}}{2}+1, \\
\left(x_{1}, \ldots, x_{q-1}, \frac{1}{2}\right), & \frac{x_{q}}{2} \leq x_{q+1} \leq-\frac{x_{q}}{2}+1, \\
\left(x_{1}, \ldots, x_{q-1}, \frac{1-x_{q}+2 x_{q+1}}{2}\right), & x_{q+1} \leq \frac{x_{q}}{2} .
\end{array}\right.
$$


The boundary operator $\partial: S_{q+1}^{c}\left(I^{q}, \dot{I}^{q}\right) \rightarrow S_{q}^{c}\left(I^{q}, \dot{I}^{q}\right)$ applied to $\sigma$ satisfies

$$
\partial \sigma=(-1)^{q+1} \sigma_{1}-(-1)^{q+1} \sigma_{2}-(-1)^{q} \iota .
$$

Indeed, the face of $\sigma$ of index $(q, 0)$ is degenerated, and all other non-mentioned faces are in $\dot{I}^{q}$. The equality in the statement of Lemma 4.5 follows.

Now, since $f \perp(h \circ f)$ maps $\left(I^{q}, \dot{I}^{q}\right)$ into $(\mathcal{L} \cap X, *)$, this lemma implies

$$
\left[(f \perp(h \circ f))_{q}(\iota)\right]_{\mathcal{L} \cap X}^{c}=\left[(f \perp(h \circ f))_{q}\left(-\sigma_{1}+\sigma_{2}\right)\right]_{\mathcal{L} \cap X}^{c},
$$

and since

$$
(f \perp(h \circ f)) \circ \sigma_{1}=f \quad \text { and } \quad(f \perp(h \circ f)) \circ \sigma_{2}=h \circ f,
$$

one immediately sees that

$$
\left[(f \perp(h \circ f))_{q}\left(-\sigma_{1}+\sigma_{2}\right)\right]_{\mathcal{L} \cap X}^{c}=\left[(h \circ f)_{q}(\iota)-f_{q}(\iota)\right]_{\mathcal{L} \cap X}^{c} .
$$

This completes the proof of (4.4) and, consequently, the proof of Lemma 4.3.

Operator $\mathrm{VAR}_{i, q}$ also satisfies the following equivariance property.

Lemma 4.6. If $\gamma \in F^{1}(\mathcal{M} \cap X, *)$ and $f \in F^{q}(\mathcal{L} \cap X, \mathcal{M} \cap X$, *), then

$$
\operatorname{VAR}_{i, q}\left(\langle\gamma\rangle_{\mathcal{M} \cap X, *} \cdot\langle f\rangle_{\mathcal{L} \cap X, \mathcal{M} \cap X, *}\right)=\langle\gamma\rangle_{\mathcal{L} \cap X, *} \cdot \operatorname{VAR}_{i, q}\left(\langle f\rangle_{\mathcal{L} \cap X, \mathcal{M} \cap X, *}\right),
$$

where equally denotes the action of $\pi_{1}(\mathcal{M} \cap X, *)$ on $\pi_{q}(\mathcal{L} \cap X, \mathcal{M} \cap X, *)$ or the action of $\pi_{1}(\mathcal{L} \cap X, *)$ on $\pi_{q}(\mathcal{L} \cap X, *)$.

Proof. Let $\gamma^{-}$be the inverse loop of $\gamma$ and let

$$
K_{\gamma^{-}}:\left(I^{q}, \dot{I}^{q}\right) \times I \rightarrow(\mathcal{L} \cap X, \mathcal{M} \cap X)
$$

be a $\gamma^{-}$-homotopy starting at $f$ (i.e., $K_{\gamma^{-}}(x, 0)=f(x)$ for every $x \in I^{q}, K_{\gamma^{-}}(x, t)=$ $\gamma^{-}(t)$ for every $x \in \dot{I}^{q} \backslash\left\{x_{q}=0\right\}$ and every $t \in I$, and $K_{\gamma^{-}}(x, t) \in \mathcal{M} \cap X$ for every $x \in\left\{x_{q}=0\right\}$ and every $\left.t \in I\right)$. Denote by $g$ the element of $F^{q}(\mathcal{L} \cap X, \mathcal{M} \cap X, *)$ defined by $g(x):=K_{\gamma^{-}}(x, 1)$. One has

$$
\langle\gamma\rangle_{\mathcal{M} \cap X, *} \cdot\langle f\rangle_{\mathcal{L} \cap X, \mathcal{M} \cap X, *}=\langle g\rangle_{\mathcal{L} \cap X, \mathcal{M} \cap X, *} .
$$

Since $h$ leaves $\mathcal{M} \cap X$ pointwise fixed, the map

$$
\mathcal{K}_{\gamma^{-}}:\left(I^{q}, \dot{I}^{q}\right) \times I \rightarrow(\mathcal{L} \cap X, \mathcal{M} \cap X)
$$

defined by

$$
\mathcal{K}_{\gamma^{-}}\left(\left(x_{1}, \ldots, x_{q}\right), t\right):= \begin{cases}K_{\gamma^{-}}\left(\left(x_{1}, \ldots, x_{q-1}, 1-2 x_{q}\right), t\right), & 0 \leq x_{q} \leq \frac{1}{2} \\ h \circ K_{\gamma^{-}}\left(\left(x_{1}, \ldots, x_{q-1}, 2 x_{q}-1\right), t\right), & \frac{1}{2} \leq x_{q} \leq 1,\end{cases}
$$

is a $\gamma^{-}$-homotopy from $f \perp(h \circ f)$ to $g \perp(h \circ g)$ (this time $\mathcal{K}_{\gamma^{-}}(x, t)=\gamma^{-}(t)$ for every $\left.(x, t) \in \dot{I}^{q} \times I\right)$. In other words,

$$
\langle\gamma\rangle_{\mathcal{L} \cap X, *} \cdot \operatorname{VAR}_{i, q}\left(\langle f\rangle_{\mathcal{L} \cap X, \mathcal{M} \cap X, *}\right)=\operatorname{VAR}_{i, q}\left(\langle g\rangle_{\mathcal{L} \cap X, \mathcal{M} \cap X, *}\right) .
$$

Lemma 4.6 then follows from (4.7).

Finally, operator $\operatorname{VAR}_{i, q}$ also satisfies the following lemma.

Lemma 4.8. The image of operator $\mathrm{VAR}_{i, q}$ is contained in the kernel of the natural map $\pi_{q}(\mathcal{L} \cap X, *) \rightarrow \pi_{q}(X, *)$. 
Proof. A representative of an element of the image of $\mathrm{VAR}_{i, q}$ is of the form $f \perp(h \circ f)$ with $f \in F^{q}(\mathcal{L} \cap X, \mathcal{M} \cap X, *)$. Let $H$ be an isotopy giving rise to $h$ as in Lemma 1.1. One defines a homotopy $I^{q} \times I \rightarrow X$ from $f \perp(h \circ f)$ to the constant map equal to * by

$$
\left(\left(x_{1}, \ldots, x_{q}\right), t\right) \mapsto\left\{\begin{array}{llr}
H\left(f\left(x_{1}, \ldots, x_{q-1}, 1-2 x_{q}\right), 2 t\right), & 0 \leq t \leq \frac{1}{2}, & 0 \leq x_{q} \leq \frac{1}{2}, \\
h \circ f\left(x_{1}, \ldots, x_{q-1}, 2 x_{q}-1\right), & 0 \leq t \leq \frac{1}{2}, & \frac{1}{2} \leq x_{q} \leq 1, \\
h \circ f\left(x_{1}, \ldots, x_{q-1}, 1-2 x_{q}\right), & \frac{1}{2} \leq t \leq 1, & 0 \leq x_{q} \leq 1-t, \\
h \circ f\left(x_{1}, \ldots, x_{q-1}, 2 t-1\right), & \frac{1}{2} \leq t \leq 1, & 1-t \leq x_{q} \leq t, \\
h \circ f\left(x_{1}, \ldots, x_{q-1}, 2 x_{q}-1\right), & \frac{1}{2} \leq t \leq 1, & t \leq x_{q} \leq 1 .
\end{array}\right.
$$

By the first half of this homotopy, the lower part of the cell undergoes the monodromy $h$ while remaining attached to the upper part; at the end of this process the two half cells become opposite and the second half of the homotopy collapses them together.

Note that the natural map under consideration is an isomorphism or epimorphism within certain bounds according to the Lefschetz hyperplane section theorems. For instance, if $X=Y \backslash Z$ is non-singular and $d$ is the least (complex) dimension of the irreducible components of $Y$ not contained in $Z$, this map is an isomorphism for $q<d-1$ and an epimorphism for $q=d-1$ (cf. $\mathrm{HL}$ and GM1, GM2 ). Lemma 4.8 then implies that the homotopical variations are trivial for $q<d-1$ and that there are well-defined epimorphisms

$$
\begin{gathered}
\pi_{d-1}(\mathcal{L} \cap X, *) / \sum_{i} \operatorname{Im} \operatorname{VAR}_{i, d-1} \rightarrow \pi_{d-1}(X, *) \quad \text { if } d \geq 3, \\
\pi_{1}(\mathcal{L} \cap X, *) / \sqrt{\bigcup_{i} \operatorname{Im} \operatorname{VAR}_{i, 1}} \rightarrow \pi_{1}(X, *) \quad \text { if } d=2,
\end{gathered}
$$

where $\overline{\bigcup_{i} \operatorname{Im} \operatorname{VAR}_{i, 1}}$ denotes the normal subgroup generated by $\bigcup_{i} \operatorname{Im} \operatorname{VAR}_{i, 1}$. Also, in the special case $Y=\mathbb{C P}^{n}$, that is, for the complement $X=\mathbb{C P}^{n} \backslash Z$ of a projective variety of codimension $c$, the Lefschetz hyperplane section theorem for complements (cf. C2 ) together with the lemma give that the homotopical variations are trivial for $q<n+c-2$ and that there is a well-defined epimorphism

$$
\pi_{n+c-2}\left(\mathcal{L} \cap\left(\mathbb{C P}^{n} \backslash Z\right), *\right) / \sum_{i} \operatorname{Im} \operatorname{VAR}_{i, n+c-2} \rightarrow \pi_{n+c-2}\left(\mathbb{C P}^{n} \backslash Z, *\right)
$$

when $n+c \geq 4$ (if $c=1,\left(4.9^{\prime}\right)$ is only a special case of (4.9)).

In the more special case where $X=\mathbb{C P}^{n} \backslash H$ is the complement of a hypersurface $H$ with isolated singularities in $\mathbb{C P}^{n}$ with $n \geq 3$, epimorphism (4.9) (or equivalently $\left(4.9^{\prime}\right)$ ) is an isomorphism (cf. Section 5). Also, when $X=\mathbb{C P}^{2} \backslash C$ is the complement of a plane curve, $\mathcal{M} \cap X$ is reduced to a point, and Observation 4.2 shows that the epimorphism appearing in the second row of (4.9) coincides with the map that van Kampen's theorem asserts to be an isomorphism. We conjecture that (4.9) is an isomorphism for any non-singular quasi-projective variety $X$ and that $\left(4.9^{\prime}\right)$ is an isomorphism for any non-empty algebraic subvariety $Z$ of $\mathbb{C P}^{n}$. This would parallel the homological results contained in [C3, Theorem 5.1] which is a generalized 'second Lefschetz theorem'. 


\section{The Link BetWeEn $\mathcal{V} \mathcal{A R}_{i, n-1}$ AND $\mathrm{VAR}_{i, n-1}$}

Throughout this section, we work under Hypotheses 3.1.

Theorem 5.1. Under Hypotheses 3.1, the homotopical variation operator $\mathcal{V A R}_{i, n-1}$ of Chéniot-Libgober which is then well-defined (cf. Section 3) coincides with the generalized homotopical variation operator $\mathrm{VAR}_{i, n-1}$ (defined in Section 4).

Observe that this theorem implies that Theorem 7.1 of $[\mathrm{CL}$ is also valid with our operators instead of those of Chéniot-Libgober, in other words, epimorphism (4.9) (or equivalently $\left(4.9^{\prime}\right)$ ) is an isomorphism under Hypotheses 3.1 as we remarked at the end of Section 4.

Proof of Theorem 5.1. Consider the diagram obtained from diagram (3.2) by completing its lower row with the homomorphism

$$
\pi_{n-1}(\mathcal{L} \cap X, \mathcal{M} \cap X, *) \stackrel{\mathrm{VAR}_{i, n-1}}{\longrightarrow} \pi_{n-1}(\mathcal{L} \cap X, *)
$$

and its middle row with the homomorphism

$$
\pi_{n-1}\left(\mathcal{L}^{\prime} \cap X^{\prime}, \mathcal{M}^{\prime} \cap X^{\prime}, \bullet\right) \stackrel{\mathrm{VAR}_{i, n-1}^{\prime}}{\longrightarrow} \pi_{n-1}\left(\mathcal{L}^{\prime} \cap X^{\prime}, \bullet\right)
$$

defined from $\omega_{i}$ as $\operatorname{VAR}_{i, n-1}$ but with pencil $\mathcal{P}^{\prime}$ and the point $\bullet$ instead of pencil $\mathcal{P}$ and the point $*$. We have to show that this new diagram is commutative. But its lower square is indeed commutative since, given a geometric monodromy $h$ of $\mathcal{L} \cap X$ relative to $\mathcal{M} \cap X$ above $\omega_{i}$, there exists a geometric monodromy $h^{\prime}$ of $\mathcal{L}^{\prime} \cap X^{\prime}$ relative to $\mathcal{M}^{\prime} \cap X^{\prime}$ above $\omega_{i}$ such that $p \circ h^{\prime}=h \circ p$ (cf. [CL, Remark 4.2]). As to the upper square, it commutes thanks to Lemma 4.3.

\section{ACKNOWLEDGEMENTS}

The second author thanks the ABDUS SALAM International Centre for Theoretical Physics (ICTP) in Trieste for kind hospitality and support.

\section{REFERENCES}

[AGV] V.I. Arnol'd, S.M. Gusein-Zade, A.N. Varchenko, Singularities of differentiable maps, Vol. II, Monodromy and asymptotics of integrals (Birkhäuser Boston, Inc., Boston, MA, 1988). MR0966191 (89g:58024)

[C1] D. Chéniot, "Une démonstration du théorème de Zariski sur les sections hyperplanes d'une hypersurface projective et du théorème de van Kampen sur le groupe fondamental du complémentaire d'une courbe projective plane", Compositio Math. 27 (1973) 141-158. MR 0366922 (51:3168)

[C2] D. Chéniot, "Topologie du complémentaire d'un ensemble algébrique projectif", Enseign. Math. (2) 37 (1991) 293-402. MR1151752 (93h:14014)

[C3] D. Chéniot, "Vanishing cycles in a pencil of hyperplane sections of a non singular quasi-projective variety", Proc. London Math. Soc. (3) 72 (1996) 515-544. MR1376767 (97a:14018)

[CL] D. Chéniot and A. Libgober, "Zariski-van Kampen theorem for higher homotopy groups", J. Inst. Math. Jussieu 2 (2003) 495-527. MR2006797 (2005a:14024)

[GM1] M. Goresky and R. MacPherson, "Stratified Morse theory", Singularities, Part 1 (Arcata, $C A, 1981$ ), Proceedings of Symposia in Pure Mathematics 40 (American Mathematical Society, Providence, RI, 1983) 517-533. MR0713089 (84k:58017)

[GM2] M. Goresky and R. MacPherson, Stratified Morse theory (Springer-Verlag, New-York, 1988). MR0932724 (90d:57039)

[HL] H.A. Hamm and D.T. Lê, "Lefschetz theorems on quasi-projective varieties", Bull. Soc. Math. France 113 (1985) 123-142. MR0820315 (87i:32017) 
[HW] P.J. Hilton and S. Wylie, Homology theory - An introduction to algebraic topology (Cambridge University Press, Cambridge, 1965). MR0115161 (22:5963)

[LT] D.T. Lê and B. Teissier, "Cycles évanescents, sections planes et conditions de Whitney II", Singularities, Part 2 (Arcata, CA, 1981), Proceedings of Symposia in Pure Mathematics 40 (American Mathematical Society, Providence, RI, 1983) 65-103. MR0713238 (86c:32005)

[Li] A. Libgober, "Homotopy groups of the complements to singular hypersurfaces, II", Ann. of Math. (2) 139 (1994) 117-144. MR.1259366 (95d:14023)

[M] W.S. Massey, A basic course in algebraic topology (Graduate Texts in Mathematics 127, Springer-Verlag, New-York, 1991). MR1095046 (92c:55001)

[Sp] E.H. Spanier, Algebraic topology (Reprint of the 1966 original, Springer-Verlag, New York, 1989). MR0210112 (35:1007)

[St] N. Steenrod, The topology of fibre bundles (Princeton University Press, Princeton, 1951). MR0039258 (12:522b)

[vK] E.R. van Kampen, "On the fundamental group of an algebraic curve", Amer. J. Math. 55 (1933) 255-260.

[Wh] H. Whitney, "Tangents to an analytic variety", Ann. of Math. (2) 81 (1965) 496-549. MR.0192520 (33:745)

[Za] O. Zariski, "On the problem of existence of algebraic functions of two variables possessing a given branch curve", Amer. J. Math. 51 (1929) 305-328.

LATP, URA CNRS 225, Centre de Mathématiques et Informatique, Université de Provence, 39 rue F. Joliot-Curie, 13453 Marseille cédex 13, France

Department of Mathematics, The Abdus Salam International Centre for TheoretiCal Physics, Strada Costiera 11, 34014 Trieste, Italy 